\title{
Symptom burden in head and neck cancer: impact upon oral energy and protein intake
}

\author{
Heidi Ganzer • Riva Touger-Decker • James S. Parrott • \\ Barbara A. Murphy • Joel B. Epstein • \\ Maureen B. Huhmann
}

Received: 27 September 2011 / Accepted: 5 July 2012 / Published online: 24 July 2012

(C) Springer-Verlag 2012

\begin{abstract}
Purpose This study explored relationships between oral symptom burden (xerostomia, thick secretions, and mucosal sensitivity), energy and protein intake, and weight change over time among head and neck cancer (HNC) patients who have completed concurrent chemoradiation (CCR).

Methods Symptom burden was assessed utilizing the Vanderbilt Head and Neck Symptom Survey version 2.0. Weight change was measured from diagnosis to treatment completion, and to the early, mid, and late recovery stage. Energy and protein intake were determined utilizing 24-h diet recalls.

Results Forty-three adult patients treated for HNC enrolled in the study. Mean percentage weight loss from diagnosis to treatment completion was $7.91 \pm 4.06 \%$. Within the midrecovery stage significant inverse relationships were found between oral protein intake and xerostomia and mucosal sensitivity $(r=-0.818, p=0.012 ; r=-0.726, p=0.032$, respectively). After controlling for weight change, significant inverse relationships were found within the mid-recovery stage between oral
\end{abstract}

H. Ganzer $(\bowtie)$

Minnesota Oncology Hematology PA,

675 East Nicollet Boulevard, Suite 200,

Burnsville, MN 55337, USA

e-mail: heidi.ganzer@usoncology.com

R. Touger-Decker · J. S. Parrott • M. B. Huhmann

Department of Nutritional Sciences, School of Health Related

Professions, Division of Nutrition, New Jersey Dental School,

University of Medicine and Dentistry of New Jersey,

Newark, NJ, USA

B. A. Murphy

Vanderbilt-Ingram Cancer Center,

Nashville, TN, USA

\section{J. B. Epstein}

Division of Otolaryngology, Head and Neck Surgery City of Hope, Duarte, California and Medical Staff, Cedar-Sinai Medical Center, Los Angeles, CA, USA energy intake and xerostomia and mucosal sensitivity $(r=-0.740, p=0.046 ; r=-0.751, p=0.043$, respectively). Significant, inverse relationships were also found between oral protein intake and xerostomia and mucosal sensitivity ( $r=-0.835, p=0.019 ; r=-0.726, p=0.033$, respectively).

Conclusions Xerostomia and mucosal sensitivity were significantly related to oral energy and protein intake post-CCR in mid-recovery. Weight loss was greatest from diagnosis to treatment completion and continued through the mid-recovery stage. Assessment of oral symptom burden (xerostomia and mucosal sensitivity) and its impact on oral intake and weight post-CCR should be conducted routinely in good patient care.

Keywords Head and neck cancer - Symptom burden . Vanderbilt head and neck symptom survey $\cdot$ Weight change · Mucosal sensitivity $\cdot$ Xerostomia

\section{Introduction}

In 2010, 1.5 million Americans were diagnosed with cancer, 2-3\% of these were head and neck cancer (HNC) [1]. Although the proportion of individuals with $\mathrm{HNC}$ is small, the population is distinct with regard to the adverse acute and late oral health complications. While estimates vary, 90 $100 \%$ of patients with HNC that undergo concurrent chemoradiation (CCR) develop acute oral complications related to their cancer therapy $[2,3]$. Specific toxicities affecting oral intake include mucositis, taste changes, xerostomia, odynophagia, dysphagia, thick secretions, mucosal sensitivity, and edema [3-6]. Oral side effects of the tumor and/or its treatment may impact the ability to masticate and swallow leading to nutritional deficiencies. Furthermore, oral complications may have a profound impact on a patient's overall health and quality of life.

While the acute and adverse oral outcomes have been well defined, late effects of treatment have often gone 
unnoticed or are marginalized. To further complicate reporting and treatment, patients may be unaware of the long-term adverse outcomes associated with oral health problems, thus they may underreport them to their health care providers. While health care providers and patients may underestimate the consequences of oral health complications, it is clear that issues such as late effect pain, dry mouth, or mucosal sensitivity may negatively impact oral intake [7, 8]. Data describing the frequency and severity of oral health outcomes and its association with oral intake is lacking. This limits the clinician's ability to target potentially treatable causes for energy and nutrient deficiencies in this population. This study explored the relationships between select late effect oral symptoms (xerostomia, thick secretions, and mucosal sensitivity), with the nutritional parameters of weight change and energy/protein intake. We were particularly interested in assessing how oral symptoms and nutritional intake varied across early ( $0-3.9$ months post-CCR), mid (4.0-9.9 months post-CCR), and late recovery (10.0-24 months post-CCR).

The primary aim of this study was to determine the relationships between xerostomia, thick secretions, and mucosal sensitivity and oral energy and protein intake. We predicted that symptoms would impact adversely on oral energy and protein intake due to problems with food consistency of highprotein foods but that patients would be able to take in adequate calories. The following hypotheses were tested: there would be an inverse relationship between select symptom burden scores (xerostomia, thick secretions, and mucosal sensitivity) and oral protein intake; no relationship between select symptom burden scores and oral energy intake, and a positive relationship between select symptom burden scores and energy intake after controlling for confounders (weight change and presence of feeding tube). All hypotheses were tested within the mid-stage of recovery as this is the time when patients are transitioning from feeding tube (FT) to oral intake and are at greatest risk for dietary inadequacies secondary to oral symptom burden.

\section{Patients and methods}

This was a prospective, exploratory cross-sectional design study using a consecutive convenience sample. Forty-three adult patients with $\mathrm{HNC}$ were enrolled in the study between August 2010 and March 31, 2011 from two of Minnesota Oncology ambulatory cancer care centers. The study was approved by the US Oncology IRB and the University of Medicine and Dentistry of New Jersey IRB. Inclusion criteria were: age $\geq 18$ years, completed CCR within 24 months, no evidence of disease, and willing and able to complete study measures. After obtaining informed consent, participants completed two questionnaires: a demographic form and the Vanderbilt Head and Neck Symptom Survey tool (VHNSS 2.0). The PI weighed the participant and conducted multiple pass 24-h dietary recalls to determine energy and protein intake. Medical records were reviewed to collect clinical characteristics.

\section{Study measures}

Assessment of symptom burden

The VHNSS is specific to patients with HNC, addressing symptom burden and functional status. The tool targets symptoms such as xerostomia, thick secretions, and mucosal sensitivity, all of which may impact oral intake and nutritional status $[9,10]$. The VHNSS was designed to be utilized as a screening tool in the clinical setting targeting physical symptoms which necessitate intervention by the treating provider or health care team for patients undergoing active treatment and post-CCR. The VHNSS 2.0 tool contains 50 questions; patients were asked to rank responses from 0 to 10 with $0=$ no symptom and $10=$ severe symptoms. Composite scores were created utilizing specific questions from the VHNSS 2.0. The subset of scores was totaled and the mean of this total score was utilized. Xerostomia scores utilized questions 14-17, questions 18-21 assessed thick secretions, and questions $44-48$ assessed mucosal sensitivity.

\section{Assessment of weight change over time}

Weight (in pounds) was recorded at time of diagnosis (first visit to oncology clinic), at treatment completion, and at the study visit within the early, mid, and late recovery stages. These three time points were chosen because they represent distinct phases in nutritional recovery. During early recovery, many patients are still FT dependent, have significant symptoms due to mucositis and are still using large amounts of supplements, during mid-recovery patients are transitioning to an oral diet and decreasing their use of supplements, and during late recovery patients are attempting to deal with the effects of late toxicities on oral intake. Treatment completion weight (TCW) was weight at time of completion of cancer treatment, and weight at time of study visit was defined as study visit weight (SVW). Percentage of weight change from TCW to each stage of recovery (study visit weight, or SVW) was determined using the formula [(Weight $\mathrm{TCW}_{\mathrm{TC}}-$ Weight $\left._{\mathrm{SvW}}\right) /$ Weight $\left._{\mathrm{TCW}}\right] \times 100=\%$ Weight change.

Assessment of energy and protein intake

For participants utilizing a FT, tube feeding formula and volume were recorded on the 24-h dietary recall form. Oral energy and protein intakes were obtained via a 24-h multiple pass dietary recall. Dietary recall information was entered 
into Mypyramid.gov [11] which utilizes the United States Department of Agriculture database for analysis of energy (in calories) and protein (in grams).

Statistical analysis

Statistical analyses were performed using Statistical Package for Social Sciences 17.0 [12]. Pearson's product moment correlation was utilized to determine the relationships between select symptom burden scores (xerostomia, thick secretions, and mucosal sensitivity) and oral energy and protein intake. Partial correlations were utilized to determine the relationships between select symptom burden scores (xerostomia, thick secretions, and mucosal sensitivity) and oral energy and protein intake while controlling for the effect of weight change from TCW. A priori alpha was $p \leq 0.05$ and tests were two-tailed unless otherwise indicated.

\section{Results}

Fifty-eight subjects were recruited; of these, eight were ineligible due to recurrence of disease, two declined participation, four did not respond to the written invitation or follow-up phone call describing the study, and one scheduled a study appointment they did not keep. Forty-three participants completed the study. Patient demographics and clinical characteristics are shown in Table 1.

Energy and protein intake

Eighty-one percent ( $n=35,81.3 \%)$ of the participants were consuming only oral energy and protein intake. Energy and protein intake are shown in Table 2. Ten participants had a FT in place at the time of study visit; three participants were utilizing their FT for $100 \%$ of their nutritional intake, two were not utilizing their FT, and five were utilizing both oral and enteral routes to meet their nutritional needs. In this sample, $75.2 \%$ of energy and $77.2 \%$ of protein needs were provided via the FT. No participants within the mid-stage had a FT in place at the time of study visit. Two study participants retained their FT within the late stage.

\section{Weight change over time}

Mean weight loss from diagnosis to completion of treatment was $7.91 \%$ of body weight (Table 3 ). Participants continued to lose weight after completing therapy. Participants lost a mean of $3.35 \%$ body weight from completion of therapy to early recovery and $4.28 \%$ from completion of cancer treatment to mid-recovery. Weight loss did not cease until late recovery where the mean percentage weight change was positive $(0.54 \pm 7.71)$, indicating a weight gain.
Table 1 Patient demographic and clinical characteristics $(n=43)$

\begin{tabular}{|c|c|}
\hline \multicolumn{2}{|l|}{ Characteristic } \\
\hline \multicolumn{2}{|c|}{ Stage of recovery [number, $(\%)]$} \\
\hline Early & $15(34.9)$ \\
\hline Mid & $7(16.3)$ \\
\hline Late & $21(48.8)$ \\
\hline \multicolumn{2}{|l|}{ Age (year) } \\
\hline Mean & 60.14 \\
\hline Range & $31-80$ \\
\hline \multicolumn{2}{|l|}{ Race [number, $(\%)]$} \\
\hline Caucasian & $42(97.7)$ \\
\hline Other & $1(2.3)$ \\
\hline \multicolumn{2}{|l|}{ Sex [number, $(\%)]$} \\
\hline Male & $35(81.4)$ \\
\hline Female & $8(18.6)$ \\
\hline \multicolumn{2}{|l|}{ Site [number, $(\%)]$} \\
\hline Tonsil & $16(37.2)$ \\
\hline Tongue & $14(32.6)$ \\
\hline Nasal & $4(9.3)$ \\
\hline Larynx & $2(4.7)$ \\
\hline Hypopharynx & $2(4.7)$ \\
\hline Oropharynx & $2(4.7)$ \\
\hline Vallecula & $2(4.7)$ \\
\hline Other $^{\mathrm{a}}$ & $5(14)$ \\
\hline \multicolumn{2}{|l|}{ Stage [number, $(\%)]$} \\
\hline II or IIA & $2(4.7)$ \\
\hline III or IIIA & $12(27.9)$ \\
\hline IVA or IVB & $27(62.8)$ \\
\hline NOS & $1(2.3)$ \\
\hline Missing & $1(2.3)$ \\
\hline \multicolumn{2}{|l|}{ Radiation (IMRT) } \\
\hline Mean Gy & $6,863.14$ \\
\hline \multicolumn{2}{|c|}{ Chemotherapy $^{\mathrm{b}}$ [number, $\left.(\%)\right]$} \\
\hline Cisplatin & $40(93.0)$ \\
\hline Fluorouracil (5-FU) & $13(30.2)$ \\
\hline Taxotere & $11(25.6)$ \\
\hline Erbitux & $6(14.0)$ \\
\hline Carboplatin & $3(7.0)$ \\
\hline Taxol & $3(7.0)$ \\
\hline Xeloda & $1(2.3)$ \\
\hline \multicolumn{2}{|c|}{ Feeding tube placement [number, (\%)] } \\
\hline Yes & $37(86.0)$ \\
\hline No & $6(14.0)$ \\
\hline \multicolumn{2}{|c|}{ Tobacco use [number, $(\%)]$} \\
\hline No & $35(81.4)$ \\
\hline Yes & $8(18.6)$ \\
\hline \multicolumn{2}{|l|}{ Alcohol use } \\
\hline No & $24(55.8)$ \\
\hline Yes & $19(44.2)$ \\
\hline
\end{tabular}

${ }^{\text {a }}$ Percentage greater than $100 \%$; patients may have had more than one type of cancer

${ }^{\mathrm{b}}$ Percentage greater than $100 \%$; patients may have received more than one type of chemotherapy 
Table 2 Energy and protein intake (oral and enteral)
Italicized items indicate highest energy and protein intake

${ }^{a}$ Patients consuming energy from both oral and tube feeding $(n=5)$

${ }^{\mathrm{b}}$ Patients utilizing feeding tube for $100 \%$ of energy needs $(n=3)$

\begin{tabular}{|c|c|c|c|}
\hline Intake & Mean & $\mathrm{SD}$ & Min-max \\
\hline \multicolumn{4}{|l|}{ Energy } \\
\hline $100 \%$ Oral energy intake $(n=35)$ & $1,938.00$ & 812.02 & $444.00-3,738.00$ \\
\hline \multicolumn{4}{|l|}{ Partial energy intake $\mathrm{e}^{\mathrm{a}}$} \\
\hline Oral energy intake & 600.80 & 593.61 & $61.00-1,608.00$ \\
\hline Energy from tube feeding & $1,696.00$ & 462.42 & $1,050.00-2,250.00$ \\
\hline Total energy & $2,296.80$ & $1,300.37$ & $1,475.00-3,288.00$ \\
\hline $100 \%$ feeding tube energy intake ${ }^{b}$ & $2,366.67$ & 550.76 & $2,000.00-3,000.00$ \\
\hline \multicolumn{4}{|l|}{ Protein } \\
\hline $100 \%$ Oral protein intake $(n=35)$ & 76.66 & 34.61 & $13.00-147.00$ \\
\hline \multicolumn{4}{|l|}{ Partial protein intake $\mathrm{a}^{\mathrm{a}}$} \\
\hline Oral protein intake & 22.40 & 18.37 & $1.00-44.00$ \\
\hline Protein via tube feeding & 72.90 & 20.03 & $47.50-102.00$ \\
\hline Total protein & 95.30 & 20.91 & $59.50-113.00$ \\
\hline $100 \%$ feeding tube protein intake ${ }^{b}$ & 102.92 & 29.35 & $80.00-136.00$ \\
\hline
\end{tabular}

\section{VHNSS 2.0 symptom burden scores}

VHNSS scores are reported by stage of recovery and total sample in Table 4. Symptom burden was high in early recovery; the following had a mean score of $\geq 5$; difficulty eating certain foods, problems with dry mouth, dry mouth affecting chewing, thick secretions, mucosal sensitivity to food types and dryness, taste alterations affecting desire to eat, and types and amount of food eaten. The following symptom burden items improved consistently over time: appetite, the ability to maintain weight, ability to eat certain solid foods and liquids, thick secretions, mouth and throat pain contributing to swallowing and speaking difficulty, average level of pain overall, symptoms involving taste, and symptoms involving mucosal sensitivity. Symptoms that remained problematic for the majority of participants through late recovery were eating certain solid foods, length of time required to eat related to swallowing problems, xerostomia making it difficult to chew and swallow, taste altering foods eaten, mucosal sensitivity altering food type eaten, and mucosal sensitivity to dryness.

\section{Relationships between select symptom burden scores} and oral energy and protein intake

Relationships between oral energy and protein intakes and select symptom burden scores by stage of recovery are reported in Table 5. Analyses excluded the three participants utilizing only enteral nutrition since oral symptoms should not impact tube feeding. After controlling for weight loss, significant, inverse relationships were found between oral energy and protein intake and xerostomia and mucosal
Table 3 Weight change over time

Weight loss indicated by $(-)$ value, weight gain $(+)$ value

\begin{tabular}{lcccr}
\hline Time period & Mean & SD & Range & Median \\
\hline Diagnosis to treatment completion $(n=43)$ & & & & \\
Weight change in pounds & -15.89 & 9.77 & $-41.00 \pm 4.80$ & -14.40 \\
Percentage (\%) of weight change & -7.91 & 4.06 & $-15.59 \pm 2.81$ & -7.68 \\
Treatment completion to early recovery stage $(n=43)$ & & & -5.40 \\
Weight change in pounds & -6.50 & 8.52 & $-38.60 \pm 10.20$ & -2.78 \\
Percentage (\%) of weight change & -3.35 & 4.29 & $-14.80 \pm 8.10$ & -4.10 \\
Treatment completion to mid-recovery stage $(n=28)$ & & & -2.18 \\
Weight change in pounds & -8.78 & 13.05 & $-47.40 \pm 4.70$ & -1.80 \\
Percentage (\%) of weight change & -4.28 & 6.05 & $-21.74 \pm 3.25$ & \\
Treatment completion to late recovery stage $(n=21)$ & & & \\
Weight change in pounds & -1.28 & 14.19 & $-37.80 \pm 25.70$ & +1.02 \\
Percentage (\%) of weight change & 0.54 & 7.71 & $-14.49 \pm 17.79$ & \\
\hline
\end{tabular}


Table 4 VHNSS scores for total sample and by recovery stage

\begin{tabular}{|c|c|c|c|c|c|c|c|c|c|}
\hline \multirow[t]{2}{*}{ Stem } & $\mathrm{m}$ & \multicolumn{2}{|c|}{ Early } & \multicolumn{2}{|c|}{ Mid } & \multicolumn{2}{|c|}{ Late } & \multicolumn{2}{|c|}{$\begin{array}{l}\text { VHNSS scores } \\
\text { for total sample }\end{array}$} \\
\hline & & $N$ & Mean \pm SD & $N$ & Mean \pm SD & $N$ & Mean \pm SD & $N$ & Mean \pm SD \\
\hline 1 & I have been losing weight & 15 & $3.07 \pm 2.52$ & 7 & $0.57 \pm 0.98$ & 21 & $1.38 \pm 2.54$ & 43 & $1.84 \pm 2.50$ \\
\hline 2 & I have lost my appetite & 15 & $4.60 \pm 3.31$ & 7 & $3.43 \pm 2.23$ & 21 & $1.81 \pm 3.01$ & 43 & $3.05 \pm 3.21$ \\
\hline 3 & I have to use liquid supplements to maintain my weight & 15 & $4.33 \pm 4.25$ & 7 & $0.57 \pm 0.98$ & 21 & $1.14 \pm 3.07$ & 43 & $2.16 \pm 3.64$ \\
\hline 4 & I have trouble maintaining my weight because of swallowing problems & 15 & $4.53 \pm 4.10$ & 7 & $2.00 \pm 1.63$ & 21 & $0.95 \pm 2.01$ & 43 & $2.37 \pm 3.26$ \\
\hline 5 & I have trouble eating certain solid foods & 15 & $6.53 \pm 3.48$ & 7 & $5.57 \pm 3.05$ & 21 & $4.48 \pm 3.94$ & 43 & $5.37 \pm 3.70$ \\
\hline 6 & I have trouble drinking thin liquids & 15 & $2.87 \pm 4.02$ & 7 & $0.57 \pm 0.98$ & 21 & $0.76 \pm 1.73$ & 43 & $1.47 \pm 2.83$ \\
\hline 7 & Food gets stuck in my mouth & 15 & $3.53 \pm 3.48$ & 7 & $2.86 \pm 4.10$ & 21 & $3.86 \pm 2.90$ & 43 & $3.58 \pm 3.25$ \\
\hline 8 & Food gets stuck in my throat & 15 & $4.07 \pm 4.04$ & 7 & $4.57 \pm 2.70$ & 21 & $3.86 \pm 2.78$ & 43 & $4.05 \pm 3.20$ \\
\hline 9 & I choke or strangle on liquids & 15 & $2.00 \pm 3.36$ & 7 & $0.86 \pm 1.21$ & 21 & $0.48 \pm 0.68$ & 43 & $1.07 \pm 2.16$ \\
\hline 10 & I choke or strangle on solid foods & 15 & $2.80 \pm 3.12$ & 7 & $2.29 \pm 2.21$ & 21 & $2.86 \pm 2.83$ & 43 & $2.74 \pm 2.80$ \\
\hline 11 & I cough after I swallow & 15 & $2.40 \pm 3.07$ & 7 & $1.14 \pm 1.35$ & 21 & $2.10 \pm 2.45$ & 43 & $2.05 \pm 2.54$ \\
\hline 12 & Swallowing takes great effort & 15 & $3.47 \pm 3.27$ & 7 & $3.71 \pm 2.29$ & 21 & $1.90 \pm 1.95$ & 43 & $2.74 \pm 2.61$ \\
\hline 13 & It takes me longer to eat because of swallowing problems & 15 & $4.27 \pm 3.51$ & 7 & $6.43 \pm 3.55$ & 21 & $4.00 \pm 3.45$ & 43 & $4.49 \pm 3.51$ \\
\hline 14 & I have problems with dry mouth & 15 & $6.33 \pm 3.11$ & 7 & $6.29 \pm 3.40$ & 21 & $6.62 \pm 2.97$ & 43 & $6.47 \pm 3.02$ \\
\hline 15 & Problems with dry mouth make chewing and swallowing difficult & 15 & $5.80 \pm 3.76$ & 7 & $6.29 \pm 3.40$ & 21 & $4.95 \pm 3.23$ & 43 & $5.47 \pm 3.41$ \\
\hline 16 & Problems with dry mouth affect my ability to sleep & 15 & $4.73 \pm 3.49$ & 7 & $1.43 \pm 2.15$ & 21 & $3.95 \pm 3.34$ & 43 & $3.81 \pm 3.36$ \\
\hline 17 & Problems with dry mouth affect my ability to talk & 15 & $3.60 \pm 2.72$ & 7 & $3.43 \pm 3.31$ & 21 & $3.57 \pm 2.82$ & 43 & $3.56 \pm 2.80$ \\
\hline 18 & I have thick mucous or phlegm & 15 & $5.53 \pm 3.11$ & 7 & $4.29 \pm 3.55$ & 21 & $3.38 \pm 3.69$ & 43 & $4.28 \pm 3.53$ \\
\hline 19 & Mucous causes me to choke or gag & 15 & $3.73 \pm 3.73$ & 7 & $2.00 \pm 2.65$ & 21 & $2.00 \pm 2.45$ & 43 & $2.60 \pm 3.03$ \\
\hline 20 & Mucous causes difficulty swallowing & 15 & $3.00 \pm 3.14$ & 7 & $3.43 \pm 3.74$ & 21 & $1.90 \pm 2.66$ & 43 & $2.53 \pm 3.01$ \\
\hline 21 & Mucous causes difficulty sleeping & 15 & $3.13 \pm 3.50$ & 7 & $1.29 \pm 1.50$ & 21 & $1.52 \pm 2.79$ & 43 & $2.05 \pm 2.96$ \\
\hline 22 & I have sores in my mouth or throat that cause pain & 15 & $2.73 \pm 3.88$ & 7 & $0.14 \pm 0.38$ & 21 & $0.48 \pm 1.78$ & 43 & $1.21 \pm 2.80$ \\
\hline 23 & Mouth or throat pain causes difficulty swallowing & 15 & $4.40 \pm 3.62$ & 7 & $2.00 \pm 1.73$ & 21 & $0.57 \pm 1.50$ & 43 & $2.14 \pm 2.99$ \\
\hline 24 & Mouth or throat pain causes difficulty speaking & 15 & $3.07 \pm 3.41$ & 7 & $1.43 \pm 1.51$ & 21 & $0.95 \pm 2.04$ & 43 & $1.77 \pm 2.67$ \\
\hline 25 & My average pain level over the last week has been... & 15 & $3.40 \pm 2.80$ & 7 & $1.43 \pm 1.51$ & 21 & $1.38 \pm 2.31$ & 43 & $2.09 \pm 2.53$ \\
\hline 26 & My worst pain over the last week has been... & 15 & $4.87 \pm 3.02$ & 7 & $1.86 \pm 1.77$ & 21 & $1.76 \pm 2.79$ & 43 & $2.86 \pm 3.07$ \\
\hline 27 & The average relief from my pain medication is... & 9 & $2.78 \pm 1.92$ & 0 & $0.00 \pm 0.00$ & 3 & $4.67 \pm 0.58$ & 12 & $3.25 \pm 1.86$ \\
\hline 28 & Pain causes difficulty sleeping & 15 & $3.53 \pm 3.42$ & 7 & $1.29 \pm 2.21$ & 21 & $1.62 \pm 3.04$ & 43 & $2.23 \pm 3.15$ \\
\hline 29 & I have trouble speaking & 15 & $2.40 \pm 3.11$ & 7 & $2.29 \pm 1.70$ & 21 & $2.19 \pm 2.52$ & 43 & $2.28 \pm 2.58$ \\
\hline 30 & My voice is hoarse & 15 & $3.27 \pm 3.13$ & 7 & $2.57 \pm 2.23$ & 21 & $3.57 \pm 3.82$ & 43 & $3.30 \pm 3.32$ \\
\hline 31 & I have trouble being understood because of my speaking or hoarse voice & 15 & $2.47 \pm 3.38$ & 7 & $1.86 \pm 1.95$ & 21 & $2.62 \pm 3.01$ & 43 & $2.44 \pm 2.95$ \\
\hline 32 & I have trouble with my hearing & 15 & $3.47 \pm 3.74$ & 7 & $0.14 \pm 0.38$ & 21 & $3.19 \pm 3.78$ & 43 & $2.79 \pm 3.59$ \\
\hline 33 & My taste is altered & 15 & $6.93 \pm 3.31$ & 7 & $4.57 \pm 3.78$ & 21 & $5.10 \pm 3.42$ & 43 & $5.65 \pm 3.49$ \\
\hline 34 & I have less desire to eat due to taste changes & 15 & $6.07 \pm 3.88$ & 7 & $4.71 \pm 3.59$ & 21 & $2.76 \pm 3.37$ & 43 & $4.23 \pm 3.82$ \\
\hline 35 & My taste changes have altered the food I choose to eat & 15 & $7.33 \pm 3.33$ & 7 & $4.86 \pm 3.53$ & 21 & $4.24 \pm 3.51$ & 43 & $5.42 \pm 3.66$ \\
\hline 36 & Taste changes have decreased amount of food I eat & 15 & $6.53 \pm 3.60$ & 7 & $4.86 \pm 3.80$ & 21 & $2.52 \pm 2.99$ & 43 & $4.30 \pm 3.75$ \\
\hline 37 & My sense of smell has changed & 15 & $3.07 \pm 3.53$ & 7 & $0.71 \pm 1.25$ & 21 & $1.86 \pm 2.69$ & 43 & $2.09 \pm 2.92$ \\
\hline 38 & I have altered what I eat due to a change in sense of smell & 15 & $2.80 \pm 3.32$ & 7 & $0.43 \pm 1.13$ & 21 & $0.76 \pm 1.48$ & 43 & $1.42 \pm 2.44$ \\
\hline 39 & I have difficulty chewing because of my teeth or dentures & 15 & $1.87 \pm 3.16$ & 7 & $0.86 \pm 2.27$ & 20 & $0.75 \pm 1.92$ & 42 & $1.17 \pm 2.48$ \\
\hline 40 & My teeth are sensitive to hot, cold, or sweet food & 13 & $3.31 \pm 3.66$ & 7 & $2.43 \pm 3.55$ & 18 & $1.50 \pm 1.95$ & 38 & $2.29 \pm 2.97$ \\
\hline 41 & My teeth feel looser & 13 & $0.54 \pm 1.39$ & 7 & $0.00 \pm 0.00$ & 18 & $0.00 \pm 0.00$ & 38 & $0.18 \pm 0.83$ \\
\hline 42 & My teeth are cracking or chipping & 13 & $0.08 \pm 0.28$ & 7 & $0.00 \pm 0.00$ & 18 & $0.89 \pm 1.84$ & 38 & $0.45 \pm 1.33$ \\
\hline 43 & I have trouble with my dentures & 2 & $4.00 \pm 2.83$ & 0 & $0.00 \pm 0.00$ & 5 & $2.40 \pm 3.91$ & 7 & $2.86 \pm 3.48$ \\
\hline 44 & I have a burning sensation in the lining of my mouth and throat & 15 & $2.33 \pm 3.29$ & 7 & $1.14 \pm 1.07$ & 21 & $1.19 \pm 2.23$ & 43 & $1.58 \pm 2.54$ \\
\hline 45 & $\begin{array}{l}\text { The lining of my mouth and throat is sensitive to spicy, } \\
\text { hot, or acidic food }\end{array}$ & 15 & $6.07 \pm 3.59$ & 7 & $5.57 \pm 4.31$ & 21 & $4.43 \pm 3.63$ & 43 & $5.19 \pm 3.72$ \\
\hline 46 & The lining of my mouth and throat is sensitive to dryness & 15 & $6.87 \pm 2.17$ & 7 & $5.14 \pm 3.48$ & 21 & $4.57 \pm 3.63$ & 43 & $5.47 \pm 3.27$ \\
\hline
\end{tabular}


Table 4 (continued)

\begin{tabular}{|c|c|c|c|c|c|c|c|c|c|}
\hline \multirow[t]{2}{*}{ Stem } & & \multicolumn{2}{|c|}{ Early } & \multicolumn{2}{|c|}{ Mid } & \multicolumn{2}{|c|}{ Late } & \multicolumn{2}{|c|}{$\begin{array}{l}\text { VHNSS scores } \\
\text { for total sample }\end{array}$} \\
\hline & & $N$ & Mean \pm SD & $N$ & Mean \pm SD & $N$ & Mean \pm SD & $N$ & Mean \pm SD \\
\hline 47 & Burning pain in the lining of my mouth and throat changes what I eat & 15 & $3.53 \pm 3.78$ & 7 & $2.71 \pm 2.56$ & 21 & $1.90 \pm 2.98$ & 43 & $2.60 \pm 3.24$ \\
\hline 48 & $\begin{array}{l}\text { Burning pain in the lining of my mouth and throat prevents me from } \\
\text { brushing my teeth }\end{array}$ & 15 & $2.40 \pm 3.38$ & 7 & $0.00 \pm 0.00$ & 21 & $0.24 \pm 0.54$ & 43 & $0.95 \pm 2.26$ \\
\hline 49 & I have limitation in the ability to open or move my jaw & 15 & $1.40 \pm 2.23$ & 7 & $2.43 \pm 2.88$ & 21 & $1.43 \pm 2.60$ & 43 & $1.58 \pm 2.49$ \\
\hline 50 & I have limitation in the ability to move my neck and shoulders & 15 & $1.27 \pm 2.31$ & 7 & $1.29 \pm 2.36$ & 21 & $3.14 \pm 3.50$ & 43 & $2.19 \pm 3.05$ \\
\hline
\end{tabular}

Italicized items indicate the highest and lowest five scores noted by study participants

sensitivity in the mid-recovery stage. Though the effect size of the relationship between thick secretions and oral energy and protein intake was large $(r \geq 0.5)$, the relationship did not reach statistical significance [13].

\section{Discussion}

The results of the current study demonstrated that symptom burden as measured by the VHNSS 2.0 was high during early recovery, improved over time, but remained problematic throughout the late stage of recovery thus confirming the results of Cooperstein et al. [10]. We then assessed the impact of symptom burden on oral energy and protein intake. The study focused on three specific symptoms; xerostomia, thick secretions, and mucosal sensitivity as these symptoms have the potential to affect the amount and type of food eaten. The authors recognize that oral symptoms are often interrelated and may be the result of disease process, treatment, or a combination of both. The VHNSS 2.0 addresses both symptom burden and functional status. Utilizing subsets of data from composite scores allowed specific data analyses as they related to xerostomia, thick secretions, and mucosal sensitivity. Mid-recovery was chosen as the time point for assessment since this is a period when patients are transitioning to an oral diet. Results demonstrated that both xerostomia and mucosal sensitivity significantly impacted oral energy and oral protein intakes in participants within the mid-stage of recovery (4.0-
Table 5 Correlations between select symptom burden scores and oral energy and protein intake by recovery stage (bivariate and controlling for weight change)

${ }^{\mathrm{a} C}$ Correlation after controlling for weight change

\begin{tabular}{|c|c|c|c|c|c|}
\hline \multirow[t]{2}{*}{ Stage } & \multirow[t]{2}{*}{ Select symptom burden scores } & \multicolumn{2}{|c|}{ Oral energy intake } & \multicolumn{2}{|c|}{ Oral protein intake } \\
\hline & & $r$ & $p$ & $r$ & $p$ \\
\hline \multirow[t]{6}{*}{ Early recovery stage $(n=13)$} & \multirow[t]{2}{*}{ Dry mouth } & -0.261 & 0.194 & -0.253 & 0.203 \\
\hline & & $-0.310^{\mathrm{a}}$ & 0.164 & $-0.322^{\mathrm{a}}$ & 0.153 \\
\hline & \multirow[t]{2}{*}{ Thick phlegm } & -0.394 & 0.091 & -0.382 & 0.099 \\
\hline & & $-0.435^{\mathrm{a}}$ & 0.079 & $-0.444^{\mathrm{a}}$ & 0.074 \\
\hline & \multirow[t]{2}{*}{ Mucosal sensitivity } & -0.525 & 0.033 & -0.409 & 0.082 \\
\hline & & $-0.471^{\mathrm{a}}$ & 0.061 & $-0.322^{\mathrm{a}}$ & 0.154 \\
\hline \multirow[t]{6}{*}{ Mid-recovery stage $(n=7)$} & \multirow[t]{2}{*}{ Dry mouth } & -0.728 & 0.032 & -0.818 & 0.012 \\
\hline & & $-0.740^{\mathrm{a}}$ & 0.046 & $-0.835^{\mathrm{a}}$ & 0.019 \\
\hline & \multirow[t]{2}{*}{ Thick phlegm } & -0.584 & 0.084 & -0.576 & 0.088 \\
\hline & & $-0.532^{\mathrm{a}}$ & 0.139 & $-0.524^{\mathrm{a}}$ & 0.143 \\
\hline & \multirow[t]{2}{*}{ Mucosal sensitivity } & -0.693 & 0.042 & -0.726 & 0.032 \\
\hline & & $-0.751^{\mathrm{a}}$ & 0.043 & $-0.784^{\mathrm{a}}$ & 0.033 \\
\hline \multirow[t]{6}{*}{ Late recovery stage $(n=20)$} & \multirow[t]{2}{*}{ Dry mouth } & 0.367 & 0.056 & 0.303 & 0.097 \\
\hline & & $0.312^{\mathrm{a}}$ & 0.097 & $0.226^{\mathrm{a}}$ & 0.176 \\
\hline & \multirow[t]{2}{*}{ Thick phlegm } & -0.12 & 0.307 & 0.01 & 0.484 \\
\hline & & $-0.112^{\mathrm{a}}$ & 0.324 & $0.034^{\mathrm{a}}$ & 0.445 \\
\hline & \multirow[t]{2}{*}{ Mucosal sensitivity } & 0.164 & 0.245 & 0.141 & 0.277 \\
\hline & & $0.045^{\mathrm{a}}$ & 0.427 & $-0.025^{\mathrm{a}}$ & 0.459 \\
\hline
\end{tabular}


9.9 months post-CCR) after controlling for weight change. Although there were strong, inverse relationships found between thick secretions and oral energy and oral protein intake, the findings were not significant. The failure to reach statistical significance may have been due to the small sample size. That being said, the hypothesis that there would be an inverse relationship between select symptom burden scores and oral protein intake within the mid-recovery stage was accepted for xerostomia and mucosal sensitivity and rejected for thick secretions.

We failed to demonstrate any relationship between xerostomia, mucosal sensitivity, and thick secretions and oral energy intake. Thus, the hypothesis that there would be no relationship between select symptom burden scores and oral energy intake was accepted. Despite this, it should be noted that there were strong inverse correlations between oral energy intake and all three symptom burden scores and the failure to reach significance may be related to the small sample size. The study clearly shows that participants continued to lose weight through mid-recovery. Oral symptom burden is a likely cause. Further study of the relationship between symptom burden and energy intake with a larger sample size is indicated.

Lastly, there was a strong significant, inverse relationship between oral energy intake and xerostomia and mucosal sensitivity when controlling for weight change. In addition, there was a strong inverse correlation between oral energy and thick secretions; however, this did not reach statistical significance. Thus, our hypothesis that there would be a positive relationship between select symptom burden scores and oral energy intake after controlling for confounders (weight change, presence of FT) was rejected. It should be noted that the analysis was done controlling for weight change only as no participants within the mid-recovery stage had a FT.

Patients that undergo CCR are at risk for significant mucositis and dysphagia which may require the use of a FT at some point during their treatment. Currently, no standard criteria exist for placement or timing of a FT in adults with HNC [14]. Within the current study sample, $86 \%(n=$ 37) had a FT placed. Of these $79.1 \%(n=34)$ had the FT placed prior to treatment, three had a FT placed during treatment. No participants had a FT placed post-CCR. The study findings concur with recommendations from the American Gastroenterological Association which indicate that feeding tubes should be used if the FT is anticipated to be utilized for more than 30 days, which is most often the situation for patients with $\mathrm{HNC}$ receiving treatment [14]. Prior researchers have reported that FT placement can decrease incidence of weight loss, prevent delay in treatment, and decrease hospitalizations [14-16]. Conversely, there is concern that FT placement may lead to increased dysphagia and potential long-term dependence on enteral nutrition
[17]. This concern was not substantiated in the current study as only two participants retained their FT within the late stage of recovery, one of which utilized the FT by choice versus necessity. All of the study participants were seen by and followed throughout CCR by an oncology registered dietitian and $79.1 \%$ were seen by a speech language pathologist. The majority of study participants had their FT removed within the early stage of recovery $(n=19,51.4 \%)$ and eight participants $(21.6 \%)$ had their FT removed during the mid-stage of recovery. The use of an integrative team approach may have been one factor that contributed to the low rate of long-term FT use.

A primary goal of nutrition therapy is to resume optimal oral intake post-CCR [18]; however, nutrition post-CCR may be challenging related to swallowing difficulty, xerostomia, thick secretions, and taste changes [19]. The findings of the current study suggest that mucosal sensitivity also was a barrier to oral intake post-CCR. Although study participants within the mid-recovery stage did resume oral intake, they may not yet have returned to optimal oral intake as determined by overall lower oral energy and protein intake and a greater weight loss from treatment completion when compared to the early or late stage of recovery.

$\mathrm{HNC}$ and its treatment have been associated with significant and unintentional weight loss with the average rate of weight loss from diagnosis to treatment completion estimated at 6 to $12 \%$ [20]. Weight loss within the current study was greatest from diagnosis to treatment completion and was found to be $7.91 \pm 4.06 \%$. These findings are consistent with other studies that demonstrated the greatest rate of weight loss occurred from diagnosis to treatment completion [15, 21-24].

Late effects of $\mathrm{HNC}$ treatment may result in alteration in normal tissue function [8]. Recognition of late toxicities, prevention, and management are needed. The resulting alteration in function may require patients to utilize adaptive strategies or coping mechanisms in regards to oral intake. Foods that are dry or require mastication may be especially difficult after treatment for $\mathrm{HNC}$ as it relates to xerostomia. Patients may utilize enteral nutrition or liquid protein supplements to meet estimated energy and protein needs. Results of the current study indicate that participants within the early stage of recovery were utilizing enteral nutrition or liquid oral supplements to meet energy needs more frequently than the mid- or late stage of recovery. Although not significant, there was a shift within the late recovery stage indicating a moderate positive relationship between oral energy intake and xerostomia. These findings suggest that study participants within the late recovery stage had adapted their diet to this late effect of treatment as VHNSS scores in the late recovery stage indicate that xerostomia had not resolved and remained problematic.

Within the current study, oral energy and protein intake was noticeably lower in those participants consuming a 
$100 \%$ oral diet. VHNSS scores indicate that those participants within the mid-stage had decreased their intake of oral liquid protein supplements and no participants in this stage of recovery had a FT in place, both are factors which may have contributed to the lower overall energy and protein intake. The overall lower intake within this stage suggests that patients had not made adaptive changes to their diet to include optimal oral energy and protein intake when compared to those participants utilizing partial or total enteral nutrition.

Mucosal sensitivity is commonly cited as a concern among patients that have completed treatment for $\mathrm{HNC}$, although it is underappreciated and often under reported. Mucosal sensitivity following treatment for $\mathrm{HNC}$ was first reported by Epstein and Stewart and it is described as a burning sensation that involves either the mouth or throat and may impact food intake and oral health care [25]. In their original work, Cooperstein et al. reported that nearly one half of their patients had complained about mucosal sensitivity with spicy, hot or acidic foods, and dryness that impacted diet [10]. The results of the current study are consistent with these findings. Although mean scores did improve consistently throughout all stages of recovery, mucosal sensitivity remained problematic for patients, and had not completely resolved within the late stage of recovery, as previously reported [25]. Within the current study, after controlling for weight change, significant strong inverse relationships were found between oral energy and protein intake and mucosal sensitivity, but only within the mid-stage of recovery.

Thick secretions are documented as an adverse affect of $\mathrm{HNC}$ treatment, however, data reporting the severity and functional impact and implications are lacking [10]. Cooperstein et al. found thick secretions to be a significant issue that remained problematic for $44.4 \%$ of patients that were greater than 6 months post-CCR [10] which is consistent with the current study with results indicating that scores improved consistently throughout each stage of recovery. However, the symptom remained problematic within the late stage of recovery. Of note, although not significant, within the mid-recovery stage strong, inverse relationships were found between oral energy and protein intake and thick secretions suggesting that thick secretions may be a barrier to oral energy and protein intake.

Murphy et al. suggest that patients with HNC may adapt their diets to consistencies that they can swallow; these adaptations may be advantageous or unfavorable [8]. Both positive adaptations and maladaptive changes were appreciated within the current study while recording the 24-h dietary recall. Participants often described consuming large quantities of fluid with meals to compensate for xerostomia. Conversely, maladaptive adaptations were also identified as participants reported avoiding meat or fruits and vegetables related to xerostomia or mucosal sensitivity. Maladaptive compensation may result in diets that are low in antioxidants, fiber, vitamins and minerals, and higher in fat [8]. VHNSS scores within the current study revealed that xerostomia and mucosal sensitivity were problematic for patients throughout all stages of recovery. The strong inverse relationship between oral energy and protein intake and xerostomia and mucosal sensitivity within the mid-stage suggests that patients within this stage had not made sufficient adaptations in regards to dry food or food that required mastication (i.e., meat). Murphy et al. suggest that patients who have completed CCR and are utilizing enteral nutrition are likely to receive adequate nutrition [8]; this was confirmed by the current study findings that indicated the highest energy and protein intake was within participants that had utilized partial or total enteral nutrition to meet their nutritional needs. Often during the transition from enteral to oral intake, alterations in diet may occur to compensate for late effects of treatment.

This study has several limitations including small sample size and a homogenous population. The results may have been different with a more varied ethnic population. Although detailed dietary recall was obtained on all participants, nutritional intake was assessed at one point versus several points in time. Determining an estimation of energy and protein needs for patients prior to initiation of treatment and monitoring oral intake through treatment and post-CCR may provide a more accurate representation of oral intake over time. Only the relationships between the select symptom burden scores of xerostomia, thick secretions, and mucosal sensitivity were assessed. Evaluation of the impact on oral energy and protein intake in regards to additional symptom burden scores, such as taste, may be of benefit.

This study is the first to address symptom burden as defined by the VHNSS 2.0, oral energy and protein intake, energy and protein intake via enteral nutrition, and weight change over time as it related to stage of recovery. This study clearly indicates that patients continued to lose weight through midrecovery. Oral symptom burden is a likely cause. Further study of the relationship between symptom burden and energy intake with larger sample size is clearly indicated. The outcomes indicate the need for multidisciplinary teams including medical and dental, nursing, nutrition, speech language pathologists, and social workers in providing optimal patient care.

Conflict of interest The PI has full control of all primary data.

Disclosures None

\section{References}

1. American Cancer Society: Cancer Facts \& Figures 2010 (2010) American Cancer Society. http://www.cancer.org/acs/groups/ content/@nho/documents/document/acspc-024113.pdf. Accessed May 15, 2011 
2. Rosenthal D, Mendoza T, Chambers M, Burkett S, Garden A, Hessell A, Lewin J, Ang K, Kies M, Gning I, Wang X, Cleeland C (2008) The MD anderson symptom inventory head and neck module, a patient reported outcome instrument, accurately predicts the severity of radiation induced mucositis. Int J Radiation Oncology Biol Phys 72(5):1355-1361

3. National Cancer Institute (2012) Oral Complications of Chemotherapy and Head/Neck Radiation http://www.cancer.gov/cancertopics/pdq/ supportivecare/oralcomplications/HealthProfessional/page6. Accessed May 16, 2011

4. Murphy B, Ridner S, Wells N, Dietrich M (2007) Quality of life research in head and neck cancer: a review of the current state of science. Critical Reviews in Oncology Hematology 62:251-267

5. Ravasco P, Monteiro-Grillo I, Camilo M (2003) Does nutrition influence quality of life in cancer patients undergoing radiotherapy? Radiotherapy and Oncology 67:213-220

6. Rosenthal D, Mendoza T, Chambers M, Aspers J, Gning I, Kies M, Weber R, Lewin J, Garden A, Ang K, Wang X, Cleeland C (2007) Measuring head and neck cancer symptom burden: the development and validation of the MD Anderson symptom inventory, head and neck module. Head \& Neck 29:923-931

7. List M, Pinar-Bilar S (2004) Functional outcomes in head and neck cancer. Seminars in Radiation Oncology 14(2):178-189

8. Murphy B, Gilbert J, Cmelak A, Ridner S (2007) Symptom control issues and supportive care of patients with head and neck cancers. Clinical Advances in Hematology \& Oncology 5(10):807-822

9. Murphy B, Dietrich M, Wells N, Dwyer K, Ridner S, Silver H, Gilbert J, Chung C, Cmelak A, Burkey B, Yarbrough W, Sinard R, Netterville J (2010) Reliability and validity of the vanderbilt head and neck symptom survey: a tool to assess symptom burden in patients treated with chemoradiation. Head Neck 32(1):26-37

10. Cooperstein E GJ, Epstein J, Dietrich M, Bond S, Ridner S, Wells N, Cmelak A, Murphy B (2010) Vanderbilt Head and Neck Symptom Survey Version 2.0: Report of the Development and initial testing of a subscale for assessment of oral health. In: American Society of Clinical Oncology Annual Meeting. pp 1-18

11. Mypyramid. http://www.mypyramid.gov/index.html. Accessed May 15, 2011

12. Statistical Package for Social Sciences: SPSS Statistics 17.0 Windows XP/Vista.

13. Cohen J (ed) (1969) Statistical power analysis for the behavioral sciences. Academic, New York

14. Locher JBJ, Carroll W, Caudell J, Keith J, Kilgore M, Ritchie C, Roth D, Tajeu G, Allison J (2011) Prophylactic percutaneous endoscopic gastrostomy tube placement in treatment of head and neck cancer: a comprehensive review and call for evidence-based medicine. Journal of Parenteral and Enteral Nutrition 35(3):365374

15. Beaver M, Matheny K, Roberts D, Myers J (2001) Predictors of weight loss during radiation therapy. Otolaryngol Head Neck Surg 125:645-648

16. Lee J, Machtay M, Unger L, Weinstein G, Weber R, Chalian A, Rosenthal D (1998) Prophylactic gastrostomy tubes in patients undergoing intensive irradiation for cancer of the head and neck. Arch Otolaryngol Head Neck Surg 124(8):871-875

17. Mekhail T, Adelstein D, Ryblckl L, Larto M, Saxton J, Lavertu P (2001) Enteral nutrition during the treatment of head and neck carcinoma: is a percutaneous endoscopic gastrostomy tube preferable to a nasogastric tube? Cancer 91(9):1785-1790

18. Kogut V, Luthringer S (eds) (2005) Nutritional issues in cancer care. Oncology Nursing Society, Pittsburgh

19. Jager-Wittenaar HDP, Vissink A, Van Oort R, Van der Laan B, Roodenburg J (2010) Malnutrition in patients treated for oral and oropharyngeal cancer - prevalence and relationship with oral symptoms: an explorative study. Support Care Cancer 19(10):1675-1683

20. Silver HDM, Murphy BA (2007) Changes in body mass, energy balance, physical function and inflammatory state in patients with locally advanced head and neck cancer treated with concurrent chemoradiation after low-dose induction chemotherapy. Head Neck 29:893-900

21. Nguyen N, Moltz C, Frank C, Vos P, Smith HJ, Karlsson U, Dutta S, Midyett FA, Barloon J, Sallah S (2004) dysphagia following chemoradiation for locally advanced head and neck cancer. Annals of Oncology 15:383-388

22. Newman L, Vieira F, Schwiezer V, Samant S, Murry T, Woodson G, Kumar P, Robbins T (1998) Eating and weight changes following chemoradiation therapy for advanced head and neck cancer. Arch Otolaryngol Head Neck Surg 124:589-592

23. van den Berg M, Rasmussen-Conrad E, Gwasara G, Krabbe P, Naber A, Merkx M (2006) A prospective study on weight loss and energy intake in patients with head and neck cancer, during diagnosis, treatment and revalidation. Clinical Nutrition 25:765-772

24. Goguen LPM, Norris C, Tishler R, Wirth L, Annino D, Gagne A, Sullivan C, Sammartino D, Haddad R (2006) Dysphagia after sequential chemoradiation therapy for advanced head and neck cancer. Otolaryngol Head Neck Surg 134:916-922

25. Epstein JSK (1993) Radiation therapy and pain in patients with head and neck cancer. Eur J Cancer Oral Oncolo 29B (3):191-199 